\title{
The influence of changes in activity-based financing on hospital readmissions for the elderly
}

\author{
JUN YIN ${ }^{1,2}$ * \\ FREDRIK A. DAHL ${ }^{1}$ \\ TERJE P. HAGEN ${ }^{2}$ \\ HILDE LURÅS ${ }^{1,3}$
}

${ }^{1}$ Helse Sør-Øst Health Services Research Centre, Akershus University Hospital, Lørenskog, Norway

${ }^{2}$ Department of Health Management and Health Economics, University of Oslo, Oslo, Norway

${ }^{3}$ Institute of Clinical Medicine, University of Oslo, Oslo, Norway

\begin{abstract}
Activity-based financing of Norwegian hospitals was implemented in 1997. An earlier study shows that when the activity-based component increases, the average length of stay for the elderly is reduced. If this reduction entails premature discharge, an increased activity-based component may have the undesirable side effect of increasing readmission rates. Yearly the Norwegian government decides the size of the activity-based component, and all hospitals face the same size. In this paper, we investigate whether the level of activity-based financing is associated with the readmission rates for acute-care patients above 70 years of age. The sample consisted of 468010 hospital admissions among elderly patients in the period from 2000 to 2007. Using repeated cross-sectional data extracted from the Norwegian Patient Registry, a Cox regression model was used to estimate factors that may influence the hazard rate of a readmission within 30 days. The overall 30-day readmission rate was $6.6 \%$. The results demonstrate that the activity-based component had no significant effect on the readmission rate. Patient-specific factors such as age, gender, diagnoses, comorbidities, as well as the time trend, were important predictors of readmission rates. We also found a statistically significant random effect of hospitals, although this effect was less substantial than the impact of patient characteristics. Our results show that the effect of the activity-based component on the readmission rate was negligible when it varied between $40 \%$ and $60 \%$.
\end{abstract}

JEL classification: H51

Key words: hospitals, elderly, activity-based financing, readmission, cox regression model

\section{Introduction}

Since July 1, 1997, Norwegian hospitals have had a mixed prospective financing system consisting of a risk-adjusted capitation component and an activity-based component (ABF). The ABF component, based on diagnosis-related groups (DRGs), has established economic incentives related to resource use (Epstein et al., 1991; Brownell et al., 1999; Yin et al., 2013). Results from the literature have shown that these incentives may encourage hospitals

* Correspondence to: Jun Yin, Helse Sør-Øst Health Services Research Centre, Akershus University Hospital, Lørenskog, Norway. E-mail: jun.yin@ahus.no

dx.doi.org/10.5617/njhe.992 
to discharge patients prematurely, leading to increased rates of readmissions (Stern and Epstein, 1985; Petersen, 2010; Lindman et al., 2012), which according to the literature are proposed to be an important indicator of patient health outcome and healthcare system performance (Anderson and Steinberg, 1984; WHO, 2005; Jencks et al., 2009; Yam et al., 2010).

Readmission rates for elderly patients are important to examine because they may influence healthcare cost and quality, and according to (Jencks et al., 2009) approximately $17 \%$ of the annual hospital payment is generated by readmissions.. In US studies of Medicare patients, 30-day readmission rates range from 8\% (Baker et al., 2004) to 21\% (Jencks et al., 2009), depending on diagnosis. A recent systematic literature which searched 34 studies published from 1966 to 2010 in the MEDLINE and EMBASE databases (van Walraven et al., 2011) reported that the median proportion of readmissions deemed avoidable was $27.1 \%$, but varied from $5 \%$ to $79 \%$.

Previous studies have found that elderly patients living in their own home have a higher probability of being readmitted to the hospital than patients living in a nursing home or an institution (Camberg et al., 1997; Heggestad, 2002), and a Canadian study reported that nursing homes reduced hospital use for patients older than 75 years of age (Shapiro et al., 1987). Thus, it appears that hospital readmission is closely connected to the organisation and resource use in the long-term care sector as well as the specific care arrangement the patients receive in their own home.

Despite their importance, little is known about recent trends in the rate of readmissions in Norway, and how the financing system affects these rates. Although several studies have provided data on readmission rates after the ABF system was implemented, none of them has controlled for case-mix or investigated the effect of the variation in the ABF component on readmission rates (Petersen, 2010; Lindman et al., 2012).

In Norway the central government decides the level of the ABF component based on political discussions in the parliament every year. Hence, the level is exogenous to the different hospitals and varies from one year to another, i.e., the level of the ABF component is identical for all hospitals. Between 2000 and 2007, the ABF changed several times, constituting between $40 \%$ and $60 \%$ of expected hospital revenues. In addition, the hospitals receive an income component from the state determined by the size and the composition of the population in the hospitals catchment area.

The current study builds on a previous study demonstrating that a $10 \%$ increase in the ABF component reduced the average length of each hospital stay by $1.3 \%$ (Yin et al., 2013). If this reduction entails premature discharge, an increased ABF component may have the undesirable side effect of increasing readmission rates. In this paper, we therefore investigate whether the level of the ABF component is associated with higher readmission rates for a group of frail, elderly patients.

We applied a Cox regression model to estimate the factors acting on the hazard rate of readmission within 30 days of discharge, and tested the hypothesis that increasing the ABF component increases the readmission rate (Heggestad, 2001, 2002).

\section{Material and methods}

\subsection{Data}

The data used in the study was taken from the Norwegian Patient Registry (NPR) and includes individual patient data from 52 acute hospitals in Norway from 2000 to 2007. The data set was repeated cross-sectional, and the patients were identifiable within a given combination of year and hospital. We limited the data set to the 14 most frequent diagnoses 
among patients 70 years and older. The ICD 10 codes for the 14 diagnoses are shown in Table 1. The variation of the ABF component from 2000 to 2007 is shown in Figure 1.

Table 1: Diagnosis group by ICD 10 codes (WHO International Classification of Diseases, http://finnkode.kith.no/)

\begin{tabular}{ll}
\hline Diagnosis group & ICD 10 code \\
\hline Pneumonia & $\mathrm{J} 13, \mathrm{~J} 15.9, \mathrm{~J} 18.0, \mathrm{~J} 18.1, \mathrm{~J} 18.9$ \\
Fracture of femur & $\mathrm{S} 72.0, \mathrm{~S} 72.1$ \\
Cerebrovascular disease & $\mathrm{I} 60-69$ \\
Acute myocardial infarction & $\mathrm{I} 21-22$ \\
Heart failure & $\mathrm{I} 50$ \\
Unspecified pain. Syncope and collapse & $\mathrm{R} 52.9, \mathrm{R} 55$ \\
Angina pectoris & $\mathrm{I} 20.1, \mathrm{I} 20.8, \mathrm{I} 20.9$ \\
Atrial fibrillation and flutter & $\mathrm{I} 48$ \\
Chronic obstructive pulmonary disease & $\mathrm{J} 44$ \\
Arthrosis & $\mathrm{M} 15-19$ \\
Certain infectious and parasitic diseases & $\mathrm{A} 01-\mathrm{B} 99$, expect A40 and A41 \\
Atherosclerosis & $\mathrm{I} 70$ \\
Cholelithiasis & $\mathrm{K} 80$ \\
Septicaemia & $\mathrm{A} 40-41$ \\
\hline
\end{tabular}

Figure 1: ABF levels for the period 2000-2007

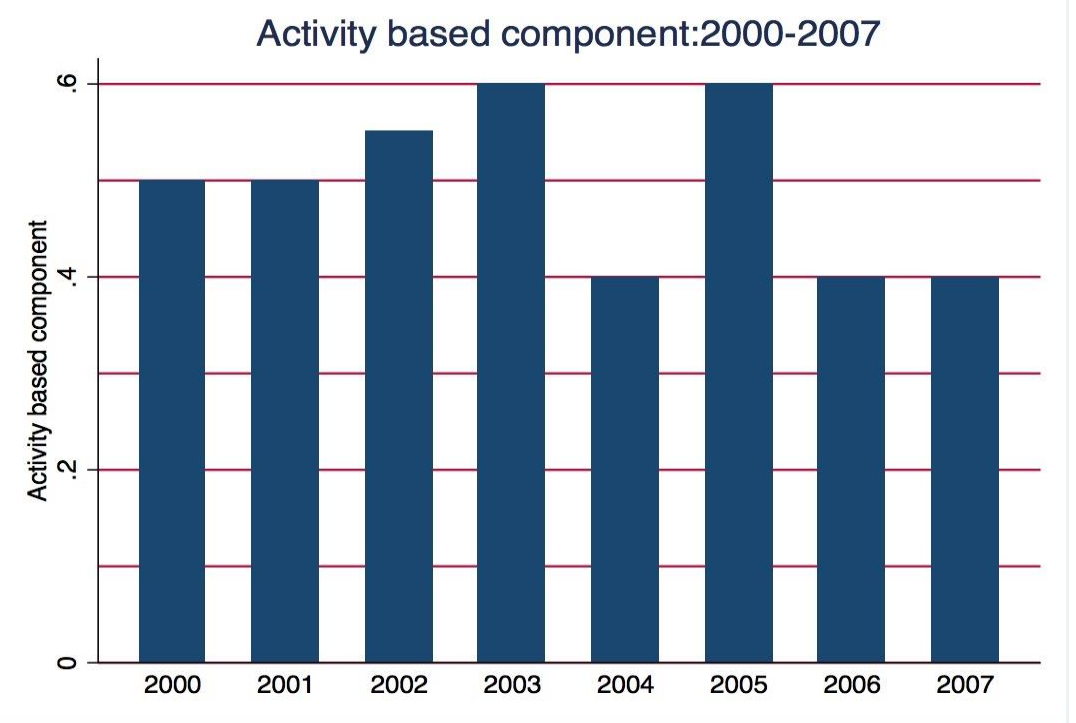

\subsection{Statistical modelling}

A patient's first admission in a year was defined as the index admission, and the event was defined as acute readmission to the same hospital within 30 days of discharge. This time frame was chosen in accordance with previous studies on readmission rates (Kerr and Byrd, 1991; Bowman, 2001; Grabowski et al., 2007). Index admissions could be either elective or acute, but for readmission only acute ones were included since elective ones were likely to be planned in advance and therefore not a result of premature discharge. The observation 
period was censored when a patient died or was readmitted for elective treatment before 30 days. The observation period was also censored at the end of the year, since readmissions in the following year could not be identified.

The level of the ABF at the time of the index admission was the main predictor, and other covariates were included primarily for the purpose of eliminating confounding effects. Although Figure 1 indicates a high level of annual variability in the ABF level, it correlates substantially with the year $(-0,43)$, and it was therefore important to include a year variable, to control for a possible trend in readmission rates. Based on the relevant literature, we chose these patient specific variables: age, gender, DRG weight, number of comorbidities, dummies for the 14 diagnoses, and a variable indicating whether the index admission was acute or elective. We also included three dummy variables to account for the patients' place of residence after the index hospitalization, respectively: (1) the patient was discharged to home (reference category); (2) the patient was discharged to institution; (3) the patient was discharged to "other places"; (Soeken et al., 1991; Corrigan and Martin, 1992; Schneider et al., 1993; Naylor et al., 1994; Rich et al., 1995; Camberg et al., 1997; Krumholz et al., 1997; Heggestad, 2002; Gillick and Steel, 1983; Shapiro et al., 1987; Kerr and Byrd, 1991; Camberg et al., 1997; Heggestad, 2002). We did not formulate specific hypotheses relating to the effect of these variables, since they were mainly included as confounders, but they might also provide findings worth discussing. To control for hospital-specific factors, we included the hospital identity as a random effect, which represents a possible positive correlation between admissions to the same hospital.

The mathematical specification of the hazard function $h(t)$ with random effects is given in Equation 1.

$$
h(t)=h_{0}(t) \exp \left(\beta_{1} A B F+\beta_{2} Y E A R+\beta_{3} X^{\prime}+\partial_{j}\right)
$$

Here, $h_{0}(t)$ is the baseline time-dependent hazard, ABF and YEAR are the level of the ABF level and the calendar year at the time of the index admission, respectively. The vector $X^{\prime}$ contains the patient specific variables, including the different care arrangements. The random effect is represented by $\partial_{j} \sim \operatorname{Gamma}\left(1, \tau^{2}\right)$, where $j$ refers to the hospital to which the patient is admitted.

A fixed effect model was also considered (Equation 2), where the random effect was substituted with a vector $H^{\prime}$ of hospital dummies.

$$
h(t)=h_{0}(t) \exp \left(\beta_{1} A B F+\beta_{2} Y E A R+\beta_{3} X^{\prime}+\beta_{4} H^{\prime}\right)
$$

The proportional hazard assumption in our Cox regression model was tested by plotting log-log survival curves. The model was estimated using STATA software (Reference Manual, Release 11, 2010, Stata Corporation, College Station, TX).

\section{Results}

\subsection{Descriptive statistics}

Table 2 provides descriptive statistics for the whole sample of 468010 individual hospital admissions included in this study, while table 3 shows descriptive statistics for the 14 different diagnoses. As can be seen the overall 30-day acute readmission rate was $6.6 \%$. 
Table 2: Characteristics of the study sample

\begin{tabular}{lllll}
\hline Variable & Mean & Std. Dev. & Min & Max \\
\hline ABF & 0.49 & 0.08 & 0.4 & 0.6 \\
$\begin{array}{l}\text { Patient level } \\
\text { Age }\end{array}$ & 80.59 & 6.38 & 70 & 108 \\
Female & 0.57 & 0.50 & 0 & 1 \\
DRG weight & 1.55 & 1.21 & 0.16 & 29.38 \\
Number of comorbidities & 2.22 & 1.76 & 0 & 7 \\
Acute index admission & 0.86 & 0.35 & 0 & 1 \\
Readmission & 0.07 & 0.25 & 0 & 1 \\
Discharged to home & 0.67 & 0.47 & 0 & 1 \\
Discharged to an institution & 0.27 & 0.44 & 0 & 1 \\
Discharged to other places & 0.06 & 0.23 & 0 & 1 \\
\hline
\end{tabular}

Table 3: Descriptive statistics by diagnosis

\begin{tabular}{llllll}
\hline Diagnosis & $\begin{array}{l}\text { Average } \\
\text { age }\end{array}$ & $\begin{array}{l}\text { Female } \\
\mathbf{( \% )}\end{array}$ & $\begin{array}{l}\text { Average } \\
\text { DRG } \\
\text { weight }\end{array}$ & $\begin{array}{l}\text { Readmis } \\
\text { sion } \\
(\boldsymbol{\%})\end{array}$ & $\begin{array}{l}\text { Percentage } \\
\text { of all } \\
\text { admissions } \\
\mathbf{( \% )}\end{array}$ \\
\hline Chronic obstructive pulmonary disease & 78.5 & 50.31 & 1.01 & 12.57 & 4.66 \\
Infectious and parasitic diseases & 80.7 & 55.62 & 1.39 & 6.30 & 7.16 \\
(excluding Septicaemia) & & & & & \\
Pneumonia & 81.6 & 48.43 & 1.51 & 7.43 & 13.09 \\
Fracture of femur & 83.5 & 75.32 & 2.40 & 3.07 & 11.61 \\
Cerebrovascular diseases & 80.8 & 53.90 & 1.63 & 4.66 & 12.00 \\
Acute myocardial infarctions & 80.4 & 47.19 & 1.44 & 12.08 & 10.28 \\
Heart failure & 82.3 & 53.21 & 1.15 & 9.55 & 7.16 \\
Angina pectoris & 79.0 & 50.66 & 0.99 & 6.40 & 6.22 \\
Unspecified pain. Syncope and collapse. & 79.9 & 60.82 & 0.42 & 4.01 & 7.27 \\
Atrial fibrillation and flutter & 79.9 & 56.95 & 0.62 & 7.73 & 5.74 \\
Arthrosis & 77.4 & 72.33 & 3.31 & 1.32 & 7.72 \\
Atherosclerosis & 79.4 & 48.95 & 2.05 & 7.30 & 3.90 \\
Cholelithiasis & 79.8 & 60.29 & 1.34 & 6.60 & 3.19 \\
Septicaemia & 80.8 & 47.66 & 2.13 & 6.47 & 2.73 \\
\hline
\end{tabular}

\subsection{Estimation results for the Cox model}

The acute readmission rate increased during the first few days after discharge from the hospital and then declined quickly (Figure 2). The parameter estimates are given in Table 4. The ABF component has no statistically significant effect on readmission rates, disproving the main hypothesis of the study. 
Table 4: $\quad$ Results of the Cox model

\begin{tabular}{|c|c|c|c|c|}
\hline Variables & Haz. Ratio & $\mathbf{Z}$ & & $\% \mathbf{C I}$ \\
\hline Activity-based component & 1.032 & 0.41 & 0.888 & 1.200 \\
\hline Age & $1.097 * * *$ & 4.57 & 1.054 & 1.142 \\
\hline $\operatorname{Age}^{\wedge} 2$ & $0.999 * * *$ & -4.62 & 0.999 & 1.000 \\
\hline Female & $0.902 * * *$ & -8.85 & 0.881 & 0.923 \\
\hline Acute & $2.389 * * *$ & 27.24 & 2.244 & 2.544 \\
\hline DRG weight & $0.930 * * *$ & -8.53 & 0.915 & 0.946 \\
\hline Comorbidity & $1.052 * * *$ & 14.27 & 1.044 & 1.059 \\
\hline Chronic obstructive pulmonary disease $\mathrm{c}^{\mathrm{c}}$ & $1.801^{* * *}$ & 23.68 & 1.715 & 1.891 \\
\hline Infectious and parasitic diseases(excluding Septicaemia) & $0.843^{* * *}$ & -5.32 & 0.792 & 0.898 \\
\hline Fracture of femur & $0.421 * * *$ & -28.52 & 0.397 & 0.447 \\
\hline Cerebrovascular & $0.620 * * *$ & -19.34 & 0.591 & 0.651 \\
\hline Acute myocardial infarctions & $1.709 * * *$ & 26.89 & 1.643 & 1.777 \\
\hline Heart failure & $1.286^{* * *}$ & 10.74 & 1.228 & 1.346 \\
\hline Angina pectoris & 1.029 & 1.01 & 0.973 & 1.089 \\
\hline Unspecified pain. Syncope and collapse & $0.545^{* * *}$ & -18.76 & 0.512 & 0.581 \\
\hline Atrial fibrillation and flutter & $1.110^{* * *}$ & 3.75 & 1.051 & 1.172 \\
\hline Arthrosis & $0.426^{* * *}$ & -15.12 & 0.382 & 0.476 \\
\hline Atherosclerosis & $1.552 * * *$ & 13.23 & 1.454 & 1.656 \\
\hline Cholelithiasis & 1.070 & 1.90 & 0.998 & 1.147 \\
\hline Septicaemia & 1.003 & 0.06 & 0.917 & 1.098 \\
\hline Year & $1.026^{* * *}$ & 8.78 & 1.020 & 1.032 \\
\hline Discharge to institution ${ }^{\mathrm{d}}$ & $1.234 * * *$ & 14.57 & 1.199 & 1.269 \\
\hline Discharge to other places & 0.990 & -0.06 & 0.933 & 1.051 \\
\hline Observations & 468010 & & & \\
\hline Number of hospitals & 52 & & & \\
\hline Theta $^{\mathrm{e}}$ & $0.019 * * *$ & & & \\
\hline 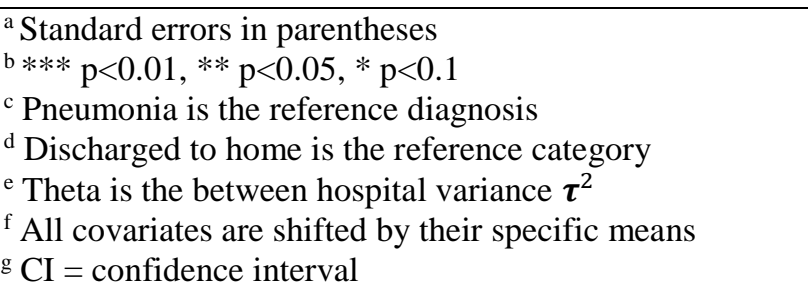 & & & & \\
\hline
\end{tabular}

Table 4 showed that almost all patient specific variables have significant impacts on readmission rates. Older patients, males and patients with larger number of comorbidities had an increased risk, while patients with higher DRG weights had reduced risk. Acute index admissions had increased readmission risk, compared to elective ones. Patients with arthrosis and fracture of the femur had much lower readmission rates than patients with other diagnoses. The hazard ratio for patients discharged to institution was 1.234 , which means that patients discharged to an institution had significantly higher readmission rates than those discharged to home.

The random effect parameter of hospitals was significant at the 0.001 level. The estimated between-hospital variance $\boldsymbol{\tau}^{2}$ was 0.019 implying a standard deviation of 0.14 . Since $\exp (0.14) \approx 1.15$, the typical hospital effect resulted in a change in the readmission rate of approximately $15 \%$. The trend variable (year) was found to have a significant positive effect on the readmission rate. The fixed effect model with 51 additional dummy 
variables for hospitals gave virtually identical estimates for the other predictors, including the ABF level, so we do not use journal space to report these.

We also estimated the model when the definition of a readmission was reduced to two, seven or 14 days, and the results were similar to those obtained for 30-day readmission.

Figure 2: The hazard function of readmissions within 30 days of discharge

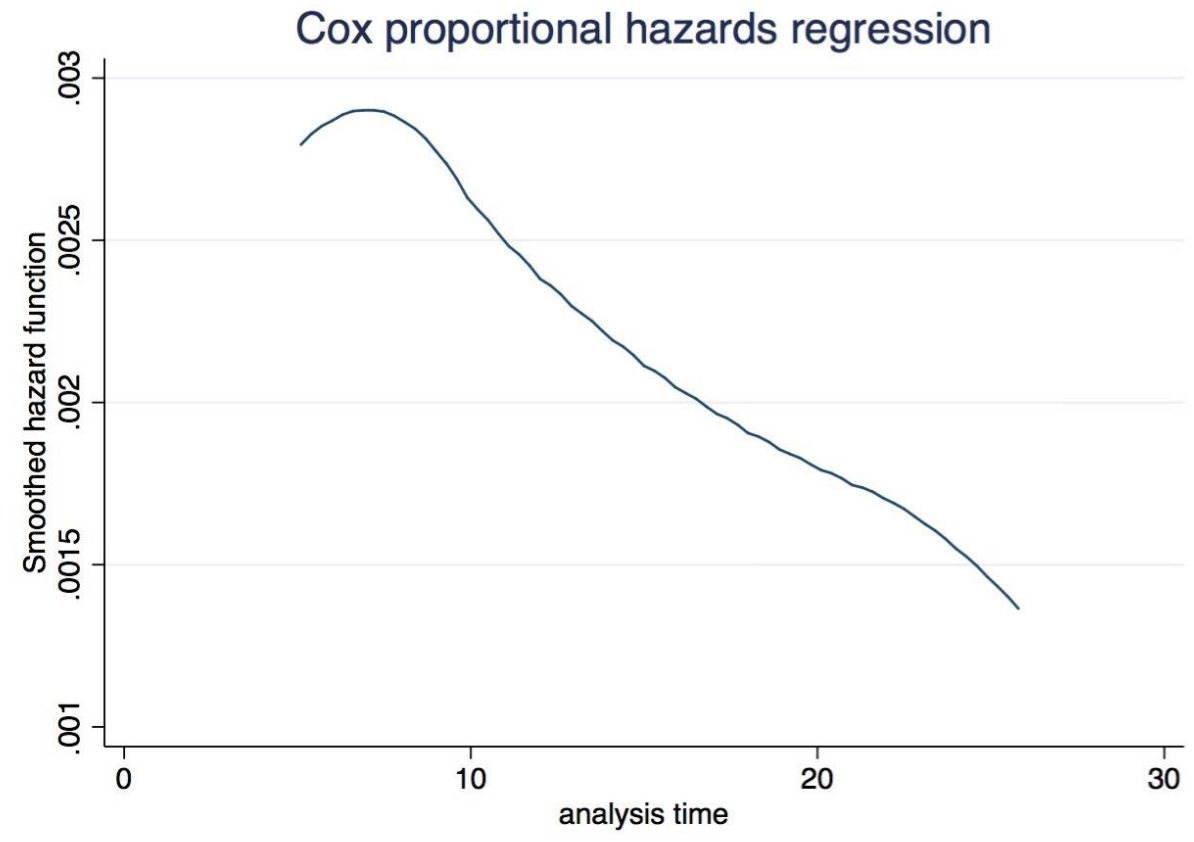

\section{Discussion}

A Norwegian study (Yin et al., 2013) demonstrated that an increased ABF level can lead to shorter hospital stays in a Norwegian sample of elderly heart disease patients. In the present study we investigate whether this has a side effect of more frequent readmissions due to premature discharge. However, we found no statistically significant effect of change in the $\mathrm{ABF}$ rates on 30-day acute readmission rates among the elderly. Compared to previous studies in the field (Heggestad, 2002; Petersen, 2010), strengths with the present study are that we control for the case-mix of patients, and that our data sample are large and almost complete, with no self-selection of patients during a seven-year time-span.

Consistent with previous studies, we found that patient-specific factors were predictors of whether a patient was readmitted to the hospital (Soeken et al., 1991; Corrigan and Martin, 1992; Schneider et al., 1993; Heggestad, 2002). Older patients and males had an increased hazard of being readmitted, as had patients with more severe illness and higher number of comorbidities. The DRG weight coefficient in the model must be interpreted as the impact of patient severity that is not captured by the patient specific variables. The contra intuitive result might stem from weaknesses in the risk adjustment. The substantially lower readmission rates for patients with the two orthopaedic diagnoses, arthrosis and fracture of the femur, can be explained by the fact that patients with these two diagnoses are often discharged from the hospital to a rehabilitation institution where they receive medical care. Patients discharged to their own home have lower readmission rates than patients discharged to an institution. Earlier studies report that readmission rates to hospitals are related to the care arrangement and long-term care resources the patient receives in their resident 
municipality (Camberg et al., 1997; Heggestad, 2002; Marshall et al., 2004). At first glance our result seems to be contrary to these findings. However, the explanation may be that the variable carries additional information on the patient's true health status, beyond the DRG weight, diagnosis, etc. Another possible explanation is that patients in institutions are more carefully monitored, so that health problems are identified more often, leading to increased readmission rates.

The random effect of hospitals was statistically significant, which indicates that there were real differences in readmission rates among hospitals, beyond the variation in the casemix. The standard deviation of the hospital level corresponds to an approximate change in the hazard rate of $15 \%$. The trend variable was found to have a significant positive effect on the readmission rate, which reflects the fact that readmission rates have been increasing annually in Norway.

Since the estimated results from the fixed effect model were virtually identical to those of the random effect model and we have no interest in the hospital-specific estimates, we prefer to use the latter, due to parsimony.

Our results and their interpretation were subject to several limitations. One limitation was that a patient may be readmitted to another hospital after discharge, which would not be included in the analysis. However, the geographical organization of Norwegian hospitals, with strictly defined catchment areas, implies that this underestimation of readmission rates most likely is negligible. Besides the purely statistical uncertainty, the diagnosis codes may not be completely reliable due to coding practices and human error. For example, a patient with a femur fracture may be encoded with a primary diagnosis of pneumonia if pneumonia occurred as a complication and required considerable resources and length of stay. However, these weaknesses are not likely to be correlated with the ABF level, so the primary conclusion of the study is robust. It is important to underline that our study do not analyse effects of introduction of $\mathrm{ABF}$ but is designed to analyse marginal changes in the ABF rate.

It is outside the scope of this paper to investigate the causes of differences in readmission rates among the hospitals. However, previous studies have shown that inadequate medical management, a lack of information from hospitals and suboptimal discharge routines may increase readmission rates (Tierney and Worth, 1995; Fernández Gracia et al., 1997). In addition, more information regarding coordination and cooperation between hospitals and municipalities, such as the distance to the hospital, nursing home characteristics etc. warrants further investigation.

\section{Conclusion}

The present analysis of a large data set of elderly patients admitted to and discharged from hospitals in Norway showed a negligible effect on readmission rates when the ABF level varied between $40 \%$ and $60 \%$. The results further showed that patient characteristics strongly influenced the readmission rates. The trend variable was found to have a significant positive effect on the readmission rate. We also found a statistically significant random effect of hospitals, although this effect was less substantial than the impact of patient characteristics. Due to the specificities of the Norwegian ABF system, the effects of the hospitals financing system on elderly patients' readmissions could be different for other countries where the institutional setting differs from the prevailing financing and organisation of the Norwegian health care system. 


\section{Acknowledgements}

The authors are grateful for the financial support from the Foundation for Health Services Research (HELTEF). In addition, Kann Inger Cathrine and Jorun Rugkåsa provided invaluable suggestions for this study. We would also like to thank the seminar participants at the Akershus University Hospital for their helpful comments and discussions.

The authors declare that they have no conflicting interests.

\section{References}

Anderson, G. F. and Steinberg, E. P. (1984). Hospital readmissions in the Medicare population. The New England journal of medicine, 311(21), pp. 1349-53.

Baker, D. W., Einstadter, D., Husak, S. S. and Cebul, R. D. (2004). Trends in postdischarge mortality and readmissions: has length of stay declined too far? Archives of internal medicine, 164(5) pp. $538-44$.

Bowman, C. E. (2001). Acute hospital admissions from nursing homes: some may be avoidable. Postgraduate Medical Journal, 77(903) pp. 40-42.

Brownell, M. D., Roos, N. P. and Burchill, C. (1999). Monitoring the impact of hospital downsizing on access to care and quality of care. Medical care, 37(6 Suppl) pp. JS135-50.

Camberg, L. C., Smith, N. E., Beaudet, M., Daley, J., Cagan, M. and Thibault, G. (1997). Discharge destination and repeat hospitalizations. Medical care, 35(8) pp. 756-67.

Corrigan, J. M. and Martin, J. B. (1992). Identification of factors associated with hospital readmission and development of a predictive model. Health services research, 27(1) pp. 81101.

Epstein, A. M., Bogen, J., Dreyer, P. and Thorpe, K. E. (1991). Trends in length of stay and rates of readmission in Massachusetts: implications for monitoring quality of care. Inquiry : a journal of medical care organization, provision and financing, 28(1) pp. 19-28.

Fernández Gracia, J., Martínez González, M. A., García Rodríguez, J., Bueno Cavanillas, A., Lardelli Claret, P. and García Martín, M. (1997). Factors associated with the incidence of hospital readmission. Medicina Clinica, 108(1) pp. 4-8.

Gillick, M. and Steel, K. (1983). Referral of patients from long-term to acute-care facilities. Journal of the American Geriatrics Society, 31(2) pp. 74-8.

Grabowski, D. C., O’Malley, A. J. and Barhydt, N. R. (2007). The costs and potential savings associated with nursing home hospitalizations. Health affairs (Project Hope), 26(6) pp. 175361.

Heggestad, T. (2001). Operating conditions of psychiatric hospitals and early readmission--effects of high patient turnover. Acta psychiatrica Scandinavica, 103(3) pp. 196-202.

Heggestad, T. (2002). Do hospital length of stay and staffing ratio affect elderly patients' risk of readmission? A nation-wide study of Norwegian hospitals. Health services research, 37(3) pp. 647-65.

Jencks, S. F., Williams, M. V and Coleman, E. A. (2009). Rehospitalizations among patients in the Medicare fee-for-service program. The New England journal of medicine, 360(14) pp. 141828.

Kerr, H. D. and Byrd, J. C. (1991). Nursing home patients transferred by ambulance to a VA emergency department. Journal of the American Geriatrics Society, 39(2) pp. 132-6.

Krumholz, H. M., Parent, E. M., Tu, N., Vaccarino, V., Wang, Y., Radford, M. J. and Hennen, J. (1997). Readmission after hospitalization for congestive heart failure among Medicare beneficiaries. Archives of internal medicine, 157(1) pp. 99-104. 
Lindman, A., Damgaard, K., Tjomsland, O. and Helgeland, J. (2012). Readmission in Norwegian hospitals-Norwegian Knowledge Center for the Health Services. Oslo.

Marshall, A. H., McClean, S. I. and Millard, P. H. (2004). Addressing bed costs for the elderly: a new methodology for modelling patient outcomes and length of stay. Health care management science, 7(1) pp. 27-33.

Naylor, M., Brooten, D., Jones, R., Lavizzo-Mourey, R., Mezey, M. and Pauly, M. (1994). Comprehensive discharge planning for the hospitalized elderly. A randomized clinical trial. Annals of internal medicine, 120(12) pp. 999-1006.

Petersen, S. O. (2010). Hospital reimbursement and readmissions. Norway 2002, 2005 and 2008. BMC Health Services Research, 10(Suppl 2) p. A14.

Rich, M. W., Beckham, V., Wittenberg, C., Leven, C. L., Freedland, K. E. and Carney, R. M. (1995). A multidisciplinary intervention to prevent the readmission of elderly patients with congestive heart failure. The New England journal of medicine, 333(18) pp. 1190-5.

Schneider, J. K., Hornberger, S., Booker, J., Davis, A. and Kralicek, R. (1993). A medication discharge planning program: measuring the effect on readmissions. Clinical nursing research, 2(1) pp. 41-53.

Shapiro, E., Tate, R. B. and Roos, N. P. (1987). Do nursing homes reduce hospital use? Medical care, 25(1) pp. 1-8.

Soeken, K. L., Prescott, P. A., Herron, D. G. and Creasia, J. (1991). Predictors of hospital readmission. A meta-analysis. Evaluation \& the health professions, 14(3) pp. 262-81.

Stern, R. S. and Epstein, A. M. (1985) Institutional responses to prospective payment based on diagnosis-related groups. Implications for cost, quality, and access. The New England journal of medicine, 312(10) pp. 621-7.

Tierney, A. J. and Worth, A. (1995). Review: readmission of elderly patients to hospital. Age and Ageing, 24(2) pp. 163-166.

Van Walraven, C., Bennett, C., Jennings, A., Austin, P. C. and Forster, A. J. (2011). Proportion of hospital readmissions deemed avoidable: a systematic review. CMAJ: Canadian Medical Association journal = journal de l'Association medicale canadienne, 183(7) pp. E391-402.

WHO (2005). Do current discharge arrangements from inpatient hospital care for the elderly reduce readmission rates, the length of inpatient stay or mortality, or improve health status?. World Health Organization.

Yam, C. H. ., Wong, L. Y., Chan, F. W. ., Wong, F. ., Leung, M. C. . and Yeoh, E. . (2010). Measuring and preventing potentially avoidable hospital readmissions: a review of the literature. Hong Kong medical journal = Xianggang yi xue za zhi / Hong Kong Academy of Medicine, 16(5) pp. 383-9.

Yin, J., Lurås, H., Hagen, T. P. and Dahl, F. A. (2013). The effect of activity-based financing on hospital length of stay for elderly patients suffering from heart diseases in Norway. BMC health services research, 13(1) p. 172.

(C) 2016 by the author(s). This article is an open access article distributed under the terms and conditions of the Creative Commons Attribution license (http://creativecommons.org/licenses/by/4.0/). 